\title{
Contribuições da Rede Brasileira de Informação em Ciências da Saúde para a construção de um repositório temático latino-americano: práticas e lições aprendidas
}

\author{
Contributions of the Brazilian Network of Information in Health Sciences \\ for the construction of a Latin American thematic repository: practices \\ and lessons learned
}

\section{Contribuciones de la Red Brasileña de Información en Ciencias de la Salud para la construcción de un repositorio temático latinoamericano: prácticas y lecciones aprendidas}

\author{
Maria de Fátima Moreira Martins | maria.correa@fiocruz.br \\ Fundação Oswaldo Cruz, Vice-Presidência de Educação, Informação e Comunicação. Rio de Janeiro, Brasil. \\ Luciana Danielli Araujo | luciana.danielli@icict.fiocruz.br \\ Fundação Oswaldo Cruz, Instituto de Comunicação e Informação Científica e Tecnológica em Saúde. Rio de Janeiro, Brasil. \\ Diones Ramos Silva | diones.ramos@icict.fiocruz.br \\ Fundação Oswaldo Cruz, Instituto de Comunicação e Informação Científica e Tecnológica em Saúde. Rio de Janeiro, Brasil. \\ Simone Faury Dib | simone.dib@icict.fiocruz.br \\ Fundação Oswaldo Cruz, Instituto de Comunicação e Informação Científica e Tecnológica em Saúde. Rio de Janeiro, Brasil.
}

\section{Resumo}

Os repositórios temáticos são utilizados como uma das principais ferramentas para a implementação do Acesso Livre no mundo e como mecanismo de preservação da memória e gestão da informação. Este trabalho busca sistematizar as estratégias utilizadas pela Rede Brasileira de Informação em Ciências da Saúde sobre os processos de cooperação e descentralização da informação técnico-científica, na perspectiva dos atores chaves envolvidos, buscando compreender os desafios, limites e possibilidades de prover acesso equitativo ao conhecimento e evidências científicas atualizadas. Aprender com os erros e acertos é fundamental para a vida de qualquer ser humano e nas instituições de ensino e pesquisa não é diferente uma vez que elas têm uma tendência a esquecer do passado. A metodologia deste estudo terá base na abordagem qualitativa, à luz de estudos de caso, por meio de aplicação de questionários semiestruturados, que tem como objetivo a coleta de informações acerca da percepção dos participantes pesquisados e de análise de conteúdo e documental. As implicações dessas lições são fundamentais para elaboração de estratégias de trabalho em rede para a construção de um repositório temático latino-americano, com a ideia de informação científica como conceito de cidadania e de comunicação pública da ciência como valor fundamental.

Palavras-chave: acesso aberto; gestão da informação; trabalho em rede; repositório temático; organização do conhecimento.

\section{Abstract}


Thematic repositories are used as one of the main tools for the implementation of Open Access in the world and as a mechanism for memory preservation and information management. This work seeks to systematize the strategies used by the Brazilian Network of Health Sciences Information on the processes of cooperation and decentralization of technical-scientific information, from the perspective of the key actors involved, seeking to understand the challenges, limits and possibilities of providing equitable access to knowledge and updated scientific evidence. Learning from mistakes and right answers is fundamental to the life of any human being and in teaching and research institutions it is no different since they have a tendency to forget the past. The methodology of this study will be based on the qualitative approach, in the light of case studies, through the application of semistructured questionnaires, whose objective is to collect information about the participants' perception and content and document analysis. The implications of these lessons are fundamental for the elaboration of networking strategies for the construction of a Latin American thematic repository, with the idea of scientific information as a concept of citizenship and public communication of science as a fundamental value.

Keywords: open access; information management; networking; thematic repository; organization of knowledge

\section{Resumen}

Los repositorios temáticos se utilizan como una de las principales herramientas para la implementación del Acceso Libre en el mundo y como mecanismo de preservación de la memoria y gestión de la información. Este trabajo busca sistematizar las estrategias utilizadas por la Red Brasileña de Información en Ciencias de la Salud sobre los procesos de cooperación y descentralización de la información técnico-científica, en la perspectiva de los actores claves involucrados, buscando comprender los desafíos, límites y posibilidades de proveer acceso equitativo al conocimiento y evidencias científicas actualizadas. Aprender con los errores y aciertos es fundamental para la vida de cualquier ser humano y en las instituciones de enseñanza e investigación no es diferente ya que tienen una tendencia a olvidar el pasado. La metodología de este estudio se basará en el abordaje cualitativo, a la luz de estudios de caso, por medio de la aplicación de cuestionarios semiestructurados, que tiene como objetivo la recolección de informaciones acerca de la percepción de los participantes investigados y de análisis de contenido y documental. Las implicaciones de estas lecciones son fundamentales para la elaboración de estrategias de trabajo en red para la construcción de un repositorio temático latinoamericano, con la idea de información científica como concepto de ciudadanía y de comunicación pública de la ciencia como valor fundamental.

Palabras claves: acceso abierto; gestión de la información; trabajo en red; el repositorio temático; organización del conocimiento.

A América Latina inicia o processo de modernização das bibliotecas através do surgimento do campo da Ciência da Informação, aplicado à pesquisa científica pelas Bibliotecas Universitárias e Especializadas. Dessa forma, o desenvolvimento tecnológico, foi considerado como área estratégica, para a produção do conhecimento nas duas primeiras décadas do século XX, após o fim da Segunda Guerra Mundial'1.

Neste sentido, entre os anos 6o-80, sob período de ditadura no Brasil, tivemos o ápice dos sistemas de informação científica, que eram apontados como estratégicos nos diversos campos de conhecimento, tais como a saúde, a energia nuclear e a agricultura. Com o desenvolvimento de coleções e sistemas de informação, surgem as redes de cooperação. O Brasil se coloca como cenário, no desenvolvimento e gestão para o acesso à informação técnico-científica em diversos campos e especialmente no campo da Saúde com Centro Latino-Americano e do Caribe de Informação em Ciências da Saúde. Organização Pan-Americana da Saúde (BIREME/OPAS).

A BIREME/OPAS surgiu do esforço entre Países, Organismos Internacionais, Órgãos Ministeriais, Universidades e Escolas Médicas, para criar a Biblioteca Regional de Medicina, inspirada a partir do modelo americano de gestão de informação científica da National Library of Medicine (NLM). Hoje é denominada 
de Centro Latino-Americano e do Caribe de Informação em Ciências da Saúde². Esta inspiração, deu-se a partir da disponibilidade do acesso às coleções de periódicos, como também, a transferência da expertise para a formação de quadros e recursos humanos estratégicos biblioteconômicos juntos aos Centros Cooperantes, no que se refere à qualificação de técnicas profissionais para fornecer estrutura e organização da Biblioteca Regional de Medicina ${ }^{3}$.

O desenvolvimento de sistemas de informação e o avanço tecnológico ocorridos na década de 80 transformaram esse período numa época de fomento para a criação de metodologias e tecnologias por meio da cooperação técnica e do trabalho em rede. Nesta perspectiva, a BIREME/OPAS cria nesse momento a Metodologia LILACS, um Sistema de Informação desenvolvido para a alimentação pela descrição e indexação de registros bibliográficos através da catalogação cooperativa.

Este desenho foi fundamental no que hoje se propõe como estrutura e gestão de diretrizes, onde o controle bibliográfico e a qualidade da informação, realizados de modo cooperativo, são fatores estruturantes para alcance da visibilidade da informação científica produzida.

A BIREME/OPAS, exerceu um papel político central para a gestão da informação técnico-científica em saúde. Seu papel como Centro Coordenador, do Sistema Regional de Informação em Ciências da Saúde, atuando no desenvolvimento de atividades cooperativas do Sistema para a manutenção dos produtos e serviços de informação, consolidou o fortalecimento das coleções e acervos das bibliotecas área para fornecer serviços como cópia de artigos científicos e treinamento aos profissionais de informação, dando origem à Rede Brasileira de Informação de Ciências da Saúde ${ }^{4,5}$. Além disso, a BIREME/OPAS, serviu como um laboratório de experimentação para o desenvolvimento de sistemas e plataformas tecnológicas quanto à gestão de fontes, produtos e serviços de Informação em Ciências da Saúde na América Latina e Caribe.

Numa visão dialógica, verifica-se a formação de uma política de informação científica que converge às políticas de saúde pública, a partir da integração do trabalho da descentralização da informação em rede como atividade funcional e estratégica. Trabalho este que é baseado em fatores metodológicos (descrição e indexação) tecnológicos (desenho dos sistemas de informação) e humanos (comportamento de busca e acesso da informação) $)^{4,6,11}$.

Diante dos processos de cooperação e descentralização da informação técnico-científica, torna-se necessário compreender os fatores que diante de desafios, limites e possibilidades são colocados hoje para atender o objetivo de prover o acesso aberto produzido pelo campo da Saúde, bem como mensurar o impacto na qualidade da informação a partir da sua disseminação e recuperação

Em 23 de setembro de 2005, a Declaração de Salvador sobre o Acesso Aberto foi assinada pelos participantes do International Seminar on Open Access, durante o $9^{\circ}$ Congresso Mundial de Informação em Saúde e Bibliotecas (ICML ${ }^{9}$ ) e $7^{\circ}$ Congresso Regional de Informação em Ciências da Saúde (CRICS7), promovidos pela BIREME, a qual destaca a importância da implantação de políticas que visem o acesso aberto à informação científica e técnica com o objetivo de promover equidades aos países em desenvolvimento10.

Assim, como questão norteadora deste trabalho destacamos, quais são as estratégias de gestão da informação técnico-científica em saúde desenvolvidas pelo trabalho em rede, que podem contribuir para a construção de repositório temático em saúde latino-americano, com foco nas questões de qualidade da informação no contexto de mudança para o acesso aberto ao conhecimento.

Partindo dessa premissa, o estudo pretende: a) identificar os principais obstáculos (resistências) e fatores que favoreceram (potencialidades) a implantação da metodologia LILACS para gestão da informação descentralizada em Ciências da Saúde; b) identificar, na percepção dos atores chaves envolvidos, as dificuldades na operacionalização das ações com foco nos objetivos e propostas pela Rede Brasileira de Informação em Ciências da Saúde em relação à realidade dos Centros Cooperantes; c) apontar a percepção dos atores chaves envolvidos, as mudanças ocorridas que favorecem ou dificultam uma nova prática no 
contexto de mudança para o acesso aberto ao conhecimento; e por fim, d) identificar percepção dos atores chaves envolvidos, ações estratégicas que superem os obstáculos identificados.

Neste aspecto, o estudo de caso de gestão e mediações da informação por meio do trabalho sócio-técnico da Rede Brasileira de Informações em Ciências da Saúde, destaca a importância da descentralização, como uma atividade funcional e estratégica tornando mais ágil o funcionamento da rede ${ }^{5}$.

Vale ressaltar, sob o ponto de vista da organização, que a descentralização auxilia no compartilhamento das informações, e nos seus processos descritivos e terminológicos. A descentralização torna-se para a rede um eixo estruturante, pois permite a equidade no que se refere à visibilidade da produção científica, bem como a agilidade no acesso e a recuperação da informação.

Nessa perspectiva, percebe-se que pela natureza interdisciplinar da ciência da informação, seu objeto de estudo é compartilhado por uma diversidade de disciplinas, como a ciência da computação, a comunicação, a linguística e especialmente a biblioteconomia. O caráter interdisciplinar permite a aproximação entre áreas que investigam a mediação dos fluxos da informação, da tecnologia, da comunicação e dos atores organizados em rede, para facilitar a democratização e o acesso à informação.

Desta forma, identificam-se elementos em comum entre a ciência da informação e a biblioteconomia, estabelecendo vínculos, na medida em que se considera a informação como objeto central sob o ponto de vista do seu desempenho social e sua preocupação com a produção de registros gráficos¹.

O tema deste estudo destaca a pesquisa sobre a gestão da informação pela Rede Brasileira de Informação em Ciências da Saúde em relação à Metodologia LILACS, para a manutenção do seu Latin American Index Medicus, a partir da percepção dos atores envolvidos pertencentes aos Centros Cooperantes que compõem a Rede.

Do ponto de vista teórico, pretende-se descrever o processo histórico apresentado por Pires-Alves ${ }^{2}$, em analisar a Rede Brasileira de Informação em Ciências para estudar sua estrutura e funcionamento, junto aos processos de cooperação e descentralização que forneceu mecanismos para o compartilhamento e visibilidade da produção técnico-científica em saúde.

A segunda abordagem teórica deste estudo referencia os conceitos de Redes de Informação trabalhado por Tomael 7 junto ao conceito de análise de Redes Sociais proposto por Marteleto ${ }^{8,12}$.

No terceiro marco, este estudo fundamenta-se por aportes da Ciência da Informação e da Saúde, abordando a seguinte questão: a descentralização na gestão da informação técnico-científica em saúde é entendida como uma questão estratégica pela Rede Brasileira de Informação em Ciências da Saúde.

A metodologia deste estudo terá base na abordagem qualitativa, à luz de estudos de caso, por meio de aplicação de questionários semiestruturados, que tem como objetivo a coleta de informações acerca da percepção dos participantes pesquisados e de análise de conteúdo e documental através da exploração do conteúdo das atas, manuais, etc. ${ }^{9,13}$

Pretende-se fazer uso da Técnica de Estudos de Caso, para analisar as estratégias de gestão da informação técnico-científica pela Rede Brasileira de Informação em Ciências da Saúde. Os participantes da pesquisa serão os profissionais de informação e as equipes gestoras que atuam na Rede Brasileira de Informação em Ciências da Saúde. Os dados obtidos serão analisados e interpretados de acordo com objetivos esperados da pesquisa.

Para análise e interpretação dos dados, pretende-se lançar a mão da Análise de Conteúdo, que por meio do encontro de macro categorias desenvolvidas no tema, propõe a sua organização em unidades de análise9 . Bastante utilizada em contextos de pesquisas de comunicação, esta metodologia parece responder bem às necessidades de organização dos dados para seu melhor entendimento.

Dessa forma, entrevistas semiestruturadas vão identificar quais são os fatores que envolvem o trabalho descentralizado, e quais são os atores chaves (profissionais da informação e equipes gestoras) atuantes nos centros cooperantes da Rede, junto a Metodologia LILACS nesses 30 anos de existência. 
A partir da produção deste estudo será iniciado a aplicação da pesquisa para obter os resultados e gerar a discussão sobre o papel do trabalho cooperativo para a formação dos repositórios temáticos a partir da consolidação do trabalho em redes de informação no campo das ciências da saúde.

\section{Referências}

1. SARACEVIC T. Ciência da Informação: origem, evolução e relações. Perspec. Ciênc. Inf. 1996 jan./jun.; $1(1): 41-62$.

2. PIRES-ALVES F. A Cooperação Internacional em Saúde na Era do Desenvolvimento: informação científica e tecnologias educacionais na formação de recursos humanos (1963-1983) (Tese de Doutorado). Rio de Janeiro, Programa de Pós-Graduaçã̃o da Casa de Oswaldo Cruz - FIOCRUZ, 2011. Disponível em: http:// www.ppghcs.coc.fiocruz.br/images/teses/tese fernandopires.pdf

3. PIRES-ALVES F. Informação científica, educação médica e políticas de saúde: a Organização PanAmericana da Saúde e a criação da Biblioteca Regional de Medicina Bireme. Ciênc. saúde coletiva 2008 jun.; 13(3):899-908. Disponível em: http://www.scielo.br/scielo.php?script=sci_arttext\&pid=S1413$\underline{81232008000300012 \& \operatorname{lng}=p t \& n r m=i s o}$

4. KRZYZANOWSKI RF. Cooperação em bibliotecas no Brasil: um panorama da década de 50 até nossos dias. RBBD. 2007 jan-jun.; 3(1):1-24.

5. ZAHER C; PACKER A. O desenvolvimento da informação em saúde na América Latina e Caribe e perspectivas futuras. Ciênc. Inf. 1993 set./dez.; 22(3): 193-200.

6. BATES MJ. The invisible substrate of Information Science. J. Am. Soc. Inf. Sci. 1999; 50 (12):1043-1050.

7. TOMAEL MI. Redes de Conhecimento. Inf. \& Inf. 2005; 10(1/2). Disponível em: http://www.uel.br/ revistas/uel/index.php/informacao/article/view/1611/1366. Acesso em: 20 ago 2015.

8. MARTELETO RM. Análise de redes sociais: aplicação nos estudos de transferência da informação. Cienc. Inf. 2001 jan./abr.; 30(1):71-81.

9. MORAES R. Análise de conteúdo. Rev. Educ. 1999; 22(37): 7-33. Disponivel em: <http://cliente.argo. com.br/ mgos/analise de conteudo moraes.html> Acesso em: 11 ago. 2015.

10. DECLARAÇÃO de Salvador sobre o acesso aberto: a perspectiva dos países em desenvolvimento [Internet]. 9th International Congress on Medical Librarianship; 7 Congresso Regional de Informação em Ciências da Saúde; International Seminar on Open Access; 20-23 setembro 2005; Salvador, BA. Salvador; 23 set. 2005.

11. ROBREDO J. Da "Internet de papel" ao fim do período que se insere entre as duas Guerras Mundiais (1919-1939). Liinc Rev. 2011;7(1):19-42.

12. MARTELETO RM. Informação, rede e redes sociais: fundamentos e transversalidades. Inf. \& Inf. 2007; 12(n. esp).

13. MINAYO MCS. (Org.). Pesquisa social: teoria, método e criatividade. 19. ed. Petrópolis: Vozes; 2001. 